Modern Physics Letters A

(c) World Scientific Publishing Company

\title{
The Elemental Composition of High-Energy Cosmic Rays: Measurements with TRACER
}

\author{
P.J. Boyle \\ Enrico Fermi Institute, The University of Chicago, 933 E. 56th Street \\ Chicago, Illinois 60637, USA. \\ boyle@uchicago.edu
}

Received (Day Month Year)

Revised (Day Month Year)

\begin{abstract}
TRACER ("Transition Radiation Array for Cosmic Energetic Radiation") is a balloon borne instrument that has been developed to directly measure the composition and energy spectra of individual heavy elements up to $10^{15} \mathrm{eV}$ particle ${ }^{-1}$. TRACER achieves a large geometric factor $\left(5 \mathrm{~m}^{2} \mathrm{sr}\right)$ through the use of a Transition Radiation Detector utilizing arrays of single wire proportional tubes. TRACER has measured the energy spectra of the elements $\mathrm{O}, \mathrm{Ne}, \mathrm{Mg}, \mathrm{Si}, \mathrm{S}, \mathrm{Ar}, \mathrm{Ca}$, and Fe. The energy spectra reach energies in excess of $10^{14} \mathrm{eV}$ particle ${ }^{-1}$ and exhibit nearly the same spectral index $(2.65$ $\pm 0.05)$ for all elements.
\end{abstract}

Keywords: Cosmic rays; diffusive shock acceleration; energy spectrum; composition; transition radiation detector

PACS Nos.: 95.55.Ym; 95.85.Ry; 98.70.Sa

\section{Introduction}

Cosmic rays arriving at Earth span an energy range from $10^{8} \mathrm{eV}$ to $10^{20} \mathrm{eV}$. Measurements of the cosmic-ray flux from experiments, both on the ground and above the atmosphere, are presented in Figure 1 and show the cosmic-ray flux falling as a near featureless power law over 30 decades. Accurate measurements of the elemental composition and individual energy spectra has, however, been an experimental challenge for many years. To date, few measurements of the energy spectra above $10^{12} \mathrm{eV}$ particle ${ }^{-1}$ have been made for individual elements. In this paper we review an effort by the TRACER group to directly measure the elemental composition of cosmic rays up to $10^{15} \mathrm{eV}$ particle ${ }^{-1}$.

The TRACER ("Transition Radiation Array for Cosmic Energetic Radiation") instrument is a balloon borne detector and probably the largest cosmic-ray detector ever flown above the atmosphere. TRACER has had three successful balloon flights since 1999, yielding an exposure of $\sim 70 \mathrm{~m}^{2}$ sr days. The observational goal of the first two flights of TRACER was to measure the individual energy spectra of the heavy elements $\mathrm{O}, \mathrm{Ne}, \mathrm{Mg}, \mathrm{Si}, \mathrm{S}, \mathrm{Ar}, \mathrm{Ca}$, and $\mathrm{Fe}$. For the third flight the instrument 
was upgraded to be sensitive to the light-medium elements B, C, and, N. Future flights of TRACER are proposed and would include measurements of the the sub-Fe elements $\mathrm{Sc}, \mathrm{Ti}, \mathrm{V}, \mathrm{Cr}$, and $\mathrm{Mn}$.

This review begins with an overview of the TRACER concept and a description of the instrument in Section 2 The analysis of the TRACER data is detailed in Section 3 and the resulting energy spectra are presented and compared with measurements from other experiments in Section 4 The measurement of the elements $\mathrm{B}, \mathrm{C}$, and sub-Fe is discussed in Section 5 .

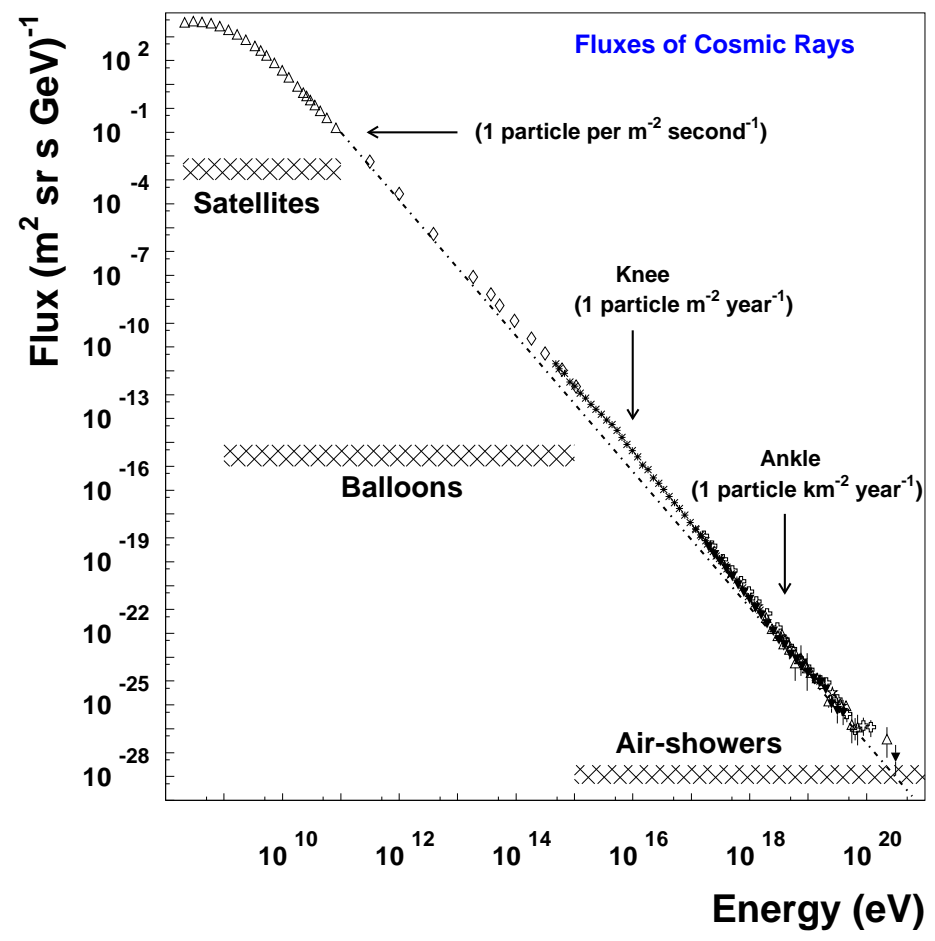

Fig. 1. All particle cosmic-ray spectrum. The hashed regions indicates the energy regions sensitive to satellites, balloon based detectors, and ground based air-shower detectors. [adapted from Cronin et al.]

\section{The TRACER Concept}

A successful cosmic-ray experiment must determine for each nucleus both the nuclear charge $Z$ and the energy $E$ or Lorentz factor $\gamma \approx E / m c^{2}$. TRACER realizes this goal by combining scintillation and Cherenkov counters to measure the charge, together with a $d E / d x$ counter and a Transition Radiation Detector (TRD) to measure the energy of individual particles up to $10^{15} \mathrm{eV}$ per particle (see Figure 2). 
The key feature on TRACER is the employment of a TRD to measure the energy or Lorentz factor of cosmic-ray nuclei up to $10^{15} \mathrm{eV}$ per particle. This application of TRD's is more challenging than their use as threshold devices in accelerator or cosmic-ray measurements 2 234], where one just wishes to discriminate between particles of the same energy but different mass (e.g. electrons and pions or protons).

Key features which distinguish TRD's from conventional energy measuring devices such as magnet spectrometers or hadronic calorimeters include: (a) a favorable area-to-weight ratio, which is important for weight-restricted-instruments on balloons or spacecraft (b) the possibility of accelerator calibrations with beams of electrons or pions at large Lorentz factors, for which beams of nuclei are not available (c) the fact that the TR-signal scales strictly with $Z^{2}$. Therefore, relative signal fluctuations decrease as $1 / Z$, making possible increasingly precise measurements for particles with higher charge. On the other hand, large signal fluctuations at low $Z$ restrict this use of TRD's to cosmic-ray nuclei heavier than helium $(Z \geq 3)$ (d) a good match between the Lorentz-factor range of a TRD $\left(400-10^{5}\right)$ and the range of energies that should be covered in direct measurements (e) the possibility of redundant measurements in a layered radiator/detector configuration (f) good energy resolution, typically of the order of $10 \%$ or better for the heavier nuclei at $\gamma \approx 1000$.

Traditionally, a TRD uses multiwire proportional chambers (MWPC's) as x-ray detectors. MWPC's require the use of a pressurized container, as employed in the Cosmic Ray Nuclei (CRN) experiment which was the first TRD flown in space [5]. The weight of such a container is large, and the need for it can be avoided by the approach taken by TRACER. For the TRACER instrument the MWPC's are replaced with layers of thin-walled single wire proportional tubes which can easily withstand internal overpressure.

\subsection{The TRACER Instrument}

Balloon borne experiments are limited in size and weight. Payloads are presently limited to $\sim 2700 \mathrm{Kg}$, of which $\sim 700 \mathrm{Kg}$ is reserved for satellite telemetry interfaces, ballast and landing gear (parachute and crush pad). TRACER is at the upper limit in terms of size and weight for a balloon payload. The employment of a TRD, without the need of a pressurized container allows for the construction of a large area detector $\left(2.06 \times 2.06 \mathrm{~m}^{2}\right)$, resulting in an overall geometric factor of $5 \mathrm{~m}^{2} \mathrm{sr}$. The individual components of TRACER are shown in Figure 2 and are summarized here. From top to bottom the instrument consists of:

1 Scintillator Counter: A plastic counter (BICRON-408) that has an active area of $2 \mathrm{~m} \mathrm{x} 2 \mathrm{~m}$ and is $0.5 \mathrm{~cm}$ thick. The counter is read out via wavelength shifter bars (BICRON 482) by 24 photomultiplier tubes (Photonis XP1910).

$2 d E / d x$ Counter: An array of 800 single wire proportional tubes. Each tube is $2 \mathrm{~m}$ long and $2 \mathrm{~cm}$ in diameter and the walls, which are made of mylar, are $127 \mu \mathrm{m}$ thick. The tubes are arranged in a total of four double layers, with each layer consisting of 100 tubes. Each double layer is orientated in 


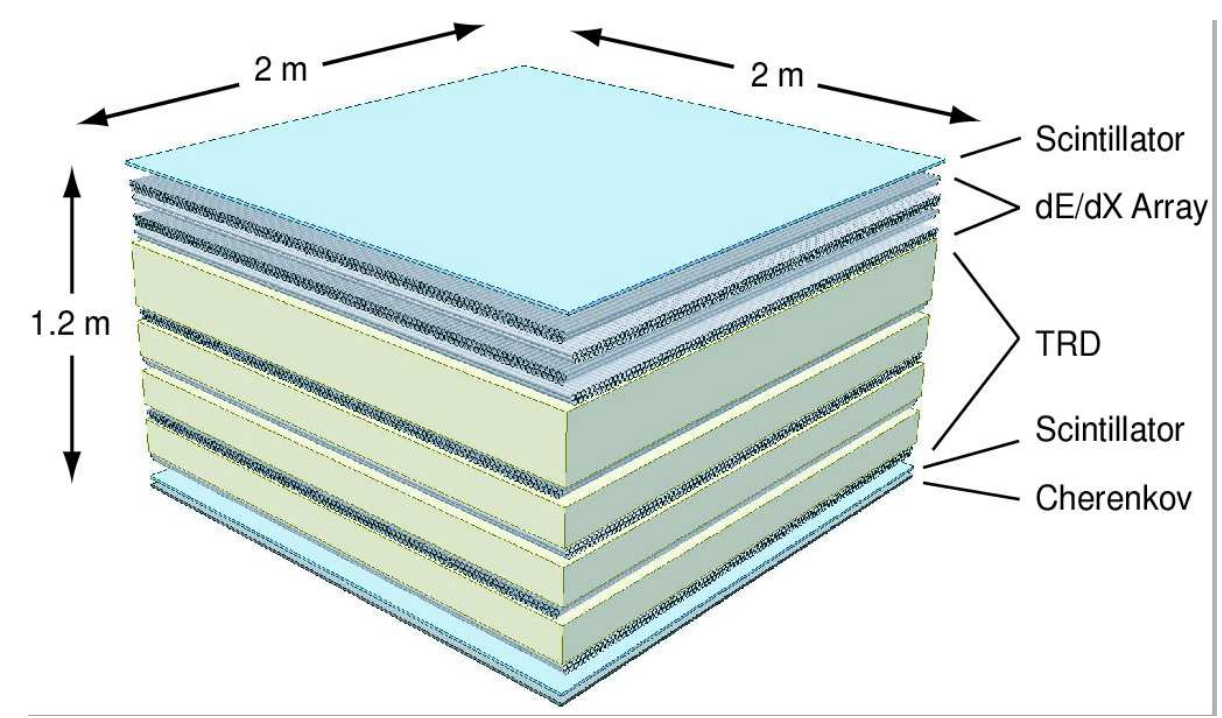

Fig. 2. Schematic diagram of the TRACER detector as flown during the Antarctic balloon campaign in 2003. [From Ave et al.]

an orthogonal direction to the one above.

3 Transition Radiation Detector: A second array of 800 single wire proportional tubes identical to the $\mathrm{dE} / \mathrm{dx}$ counter except that each double layer is preceded by blankets of plastic fiber material which act as a radiator to generate transition radiation.

4 Scintillator Counter: A second counter of identical design as the top scintillator counter is placed below the TRD.

5 Cherenkov Counter: An acrylic counter with refractive index $=1.49$ is placed at the bottom of the stack. The active area of the counter is $2 \mathrm{~m} \mathrm{x}$ $2 \mathrm{~m}$ and the thickness is $1.27 \mathrm{~cm}$. The counter is also read out, via wavelength shifting bars, by 24 PMTs.

The scintillator counters act as a trigger for the instrument and together with the Cherenkov detector measure the nuclear charge $Z$ of each individual cosmicray particle traversing the instrument. The array of all 1600 proportional tubes determines the trajectory of each cosmic-ray particle through the instrument. The energy response of the TRACER detector spans 4 decades in energy and is shown in Figure 3. This extensive energy range is accomplished by combining three complementary measurements: Cherenkov light measurement $\left(\sim 10^{11} \mathrm{eV}\right)$, the relativistic rise of the ionization signal in gas $\left(\sim 10^{11}-10^{13} \mathrm{eV}\right)$ and the measurement of transition radiation $\left(>10^{13} \mathrm{eV}\right)$. 


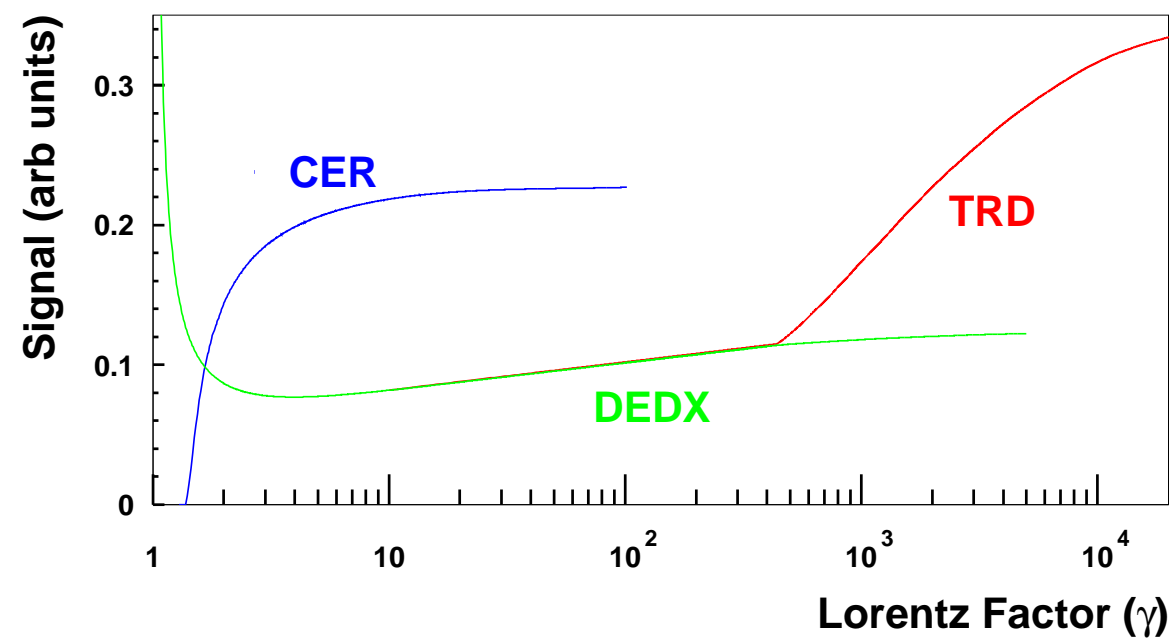

Fig. 3. Energy scale of TRACER. TRACER combines three energy measurements to cover more than 4 decades in energy. The relative amplitude of the response curves is representative of the signal $/ Z^{2}$ in each detector [From Ave et al.]

\subsection{Balloon Flights}

TRACER has had three successful flights on high altitude balloon thus far. The instrument and data have been recovered intact after each flight. A summary of the flights is as follow:

Flight I: Launch from Ft. Sumner, USA on September 20, 1999 on a 39 million cubic-foot balloon. The flight was 28 hours in duration at a float altitude between 34 and $38 \mathrm{~km}$, corresponding to a residual atmospheric depth of $4-6.5 \mathrm{~g} \mathrm{~cm}^{-2}$. This flight served as a test flight for the subsequent longer duration flights.

Flight II: Launch from Antarctica on December 12, 2003 on a 39 million cubicfoot balloon. The flight lasted 14 days at a float altitude between 36 and $39 \mathrm{~km}$, corresponding to an average residual atmospheric depth of $3.9 \mathrm{~g} \mathrm{~cm}^{-2}$. A total of $5 \times 10^{7}$ cosmic-ray particles were collected.

For the first two flights, the trigger threshold was set such that the instrument had full efficiency for the elements oxygen to iron.

Flight III: Launch from Kiruna, Sweden on July 8, 2006 on a 39 million cubicfoot balloon. The flight was limited to 4.5 days due to the lack of permission to fly over Russian territory. The float altitude was between 36 and $40 \mathrm{~km}$, corresponding to an average residual atmospheric depth of $3.5 \mathrm{~g} \mathrm{~cm}^{-2}$. For this flight, the trigger threshold was set at a signal level corresponding to a nuclear charge between lithium and beryllium. This allowed for full efficiency for the lighter elements boron, carbon, and nitrogen in addition to the heavier elements. A total of $3 \times 10^{7}$ cosmic-ray particles were collected.

The results of Flight I have been published by Gahbauer et al. (2004) [6]. The 
subsequent discussion of the analysis and results from TRACER presented in this paper pertain to Flight II. These results have been published by Ave et al. 7], where the analysis is discussed in greater detail. Analysis of data collected in Flight III is currently ongoing.

\section{Constructing an Energy Spectrum with TRACER}

The analysis of the TRACER data begins with the reconstruction of the trajectory of each cosmic-ray particle through the instrument. The accurate knowledge of the particle trajectory is essential to the analysis for two reasons; first, it permits corrections of the scintillator and Cherenkov signals due to spatial non-uniformities and zenith-angle variations, second, it makes it possible to determine accurately the energy per unit pathlength deposited by each cosmic ray traversing the proportional tubes.

\subsection{Trajectory Reconstruction}

The trajectory of cosmic rays through the instrument is reconstructed for signals recorded in the entire proportional tube array. The reconstruction follows a two step procedure. As a first estimate, the trajectory is obtained by constructing a straight line fit to the center of each of the tubes hit in an event. All possible combinations of these tubes are fit in the $\mathrm{x}$ - and $\mathrm{y}$-projections, and the combination with the minimum $\chi^{2}$ per degree of freedom and maximum number of tubes hit is kept. This procedure reconstructs the trajectories of over $95 \%$ of all cosmic-ray particles, with a lateral accuracy in the track position of $5 \mathrm{~mm}$. The trajectory is then refined using the fact that the energy deposited in each tube is proportional to the track length in that tube. Taking this fact into account, an accuracy of $2 \mathrm{~mm}$ in lateral track position is achieved, which corresponds to a $3 \%$ uncertainty in the total path length through all tubes.

\subsection{Charge Reconstruction}

The charge is determined for each cosmic-ray particle using the signals from the scintillation and Cherenkov counters. The signals are corrected for spatial nonuniformities in the counter responses using the trajectory information together with response maps recorded with muons on the ground. Figure 4 shows a correlation of the signals from the top scintillator and Cherenkov counters. Particles of constant charge are clustered along the lines, with the position on the line dependent on the primary energy. A charge histogram is then constructed through the summation along these lines of constant charge.

The resulting charge resolution is both charge and energy dependent. For oxygen nuclei $(\mathrm{Z}=8)$ the charge evolves from 0.25 at energies below $3 \mathrm{GeV}^{\mathrm{amu}}{ }^{-1}$ to 0.30 charge units at higher energies, while for iron nuclei $(Z=26)$ the resolution is 0.5 and 0.6 charge units respectively. 

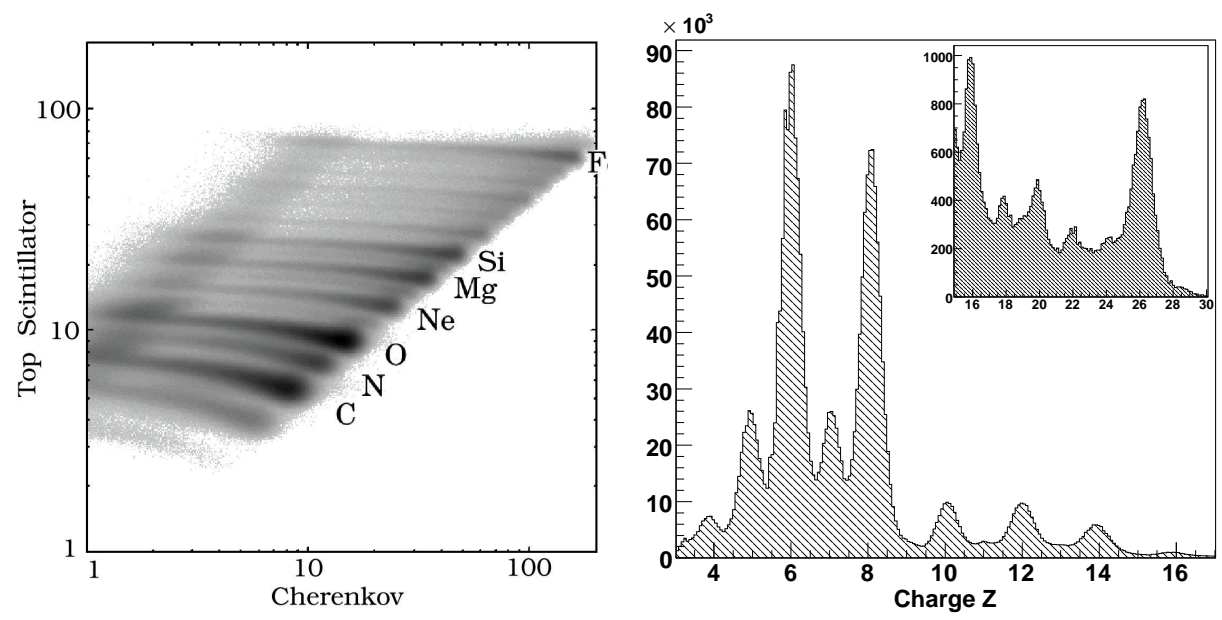

Fig. 4. Scatter plot of the signal from the scintillator and Cherenkov counters (left). A charge histogram for all events is obtained by summing along lines of constant charge (right). [From Ave et al.]

\subsection{Energy Reconstruction}

The energy of each cosmic-ray particle is obtained from the combined signals of the Cherenkov counter and of the proportional tubes. The Cherenkov counter identifies particles below minimum ionization energy $\left(3 \mathrm{GeV}_{\mathrm{amu}}{ }^{-1}\right)$, and also provides a good energy measurement for these low-energy particles (see Figure 3). Between minimum ionization energy and the onset of $\mathrm{TR}\left(400 \mathrm{GeV} a m u^{-1}\right)$, the signals in the $\mathrm{dE} / \mathrm{dx}$ counter and the TRD are the same and increase logarithmically with energy (see Figure 3). Above $400 \mathrm{GeV} \mathrm{amu} u^{-1}$, the signals from the $\mathrm{dE} / \mathrm{dx}$ counter and TRD diverge, and the rapid increase in the TR signals with particle energy provides an excellent energy measurement. However, cosmic-ray particles in this energy region are extremely rare, being less abundant than particles in the minimum ionization energy region by more than 4 orders of magnitude. To uniquely identify these rare cosmic-ray particles, it is required that particles with low-energy are identified and removed on the basis of their Cherenkov signals. To select the highest energy events, it is required that the measurement in the $\mathrm{dE} / \mathrm{dx}$ counter places these particles at an energy level well above minimum ionization energy and the presence of TR is detected. Thus the combination of the Cherenkov counter, the $\mathrm{dE} / \mathrm{dx}$ counter and the TRD is crucial for the success of the TRACER measurement at the highest energies.

Figure 5 shows a cross-correlation plot between the TRD and $\mathrm{dE} / \mathrm{dx}$ counter signals for neon nuclei $(\mathrm{Z}=10)$. Cosmic-ray particles below minimum ionization energy have been removed with the aid of the Cherenkov counter. The small black points represent particles with energies below the onset of TR. As the signals from the TRD and $\mathrm{dE} / \mathrm{dx}$ counter are similar in this energy regime, the correlation of the 


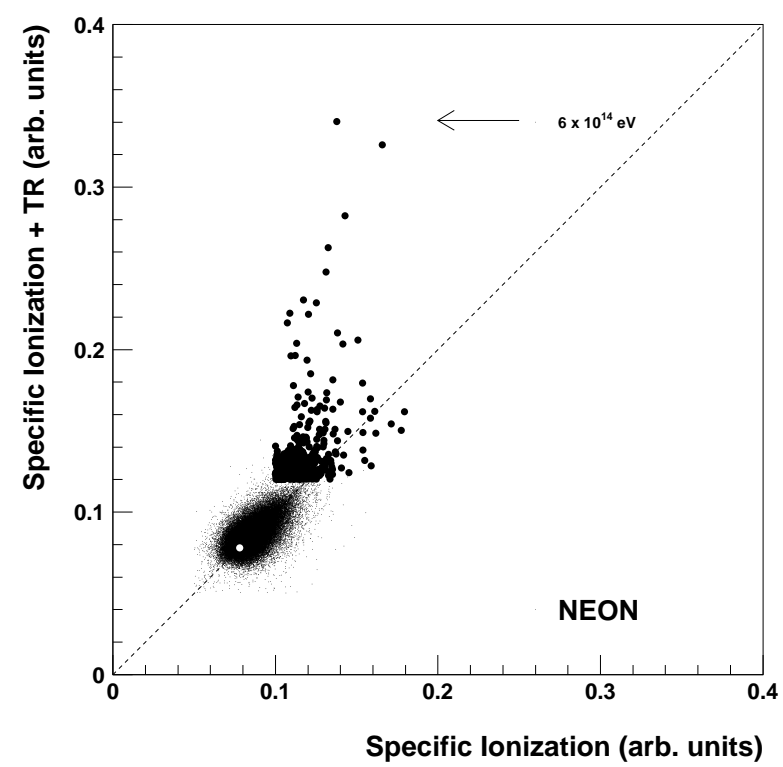

Fig. 5. Scatter plot of the signal from the TRD and the DEDX counters for neon nuclei. The highlighted points represent the highest energy events as measured with the TRD. As expected the TR events have signals in the DEDX counter which are well above the minimum ionization energy (white circle). [From Ave et al.]

signals lies along the diagonal. At the onset of TR, where the TRD and the $\mathrm{dE} / \mathrm{dx}$ signals diverge, the highest energy events show up away from the diagonal line. The off-diagonal position of these highest energy particles defines them uniquely as TR events for this selected charge. The highest energy neon nucleus in this data sample has an energy of $6 \times 10^{14} \mathrm{eV}$ particle ${ }^{-1}$.

\subsection{Absolute Intensities}

Once each cosmic-ray particle has been assigned an energy it is sorted into energy bins of width $\Delta \mathrm{E}_{i}$ and a differential energy spectrum is constructed for each elemental species. To convert from the number of events $\Delta \mathrm{N}_{i}$ in a particular energy bin $\Delta \mathrm{E}_{i}$ to an absolute flux $d N / d E(i)$ one must compute the exposure factor, effective aperture of the instrument, and the efficiency of the analysis selection and instrument response. The overall efficiency is high, with a tracking efficiency of $\sim 95 \%$, livetime $\sim 94 \%$, and charge selection efficiency $70-80 \%$ (for the more abundant elements). Further details of the reconstruction efficiencies can be found in Ave et al. 7]. 


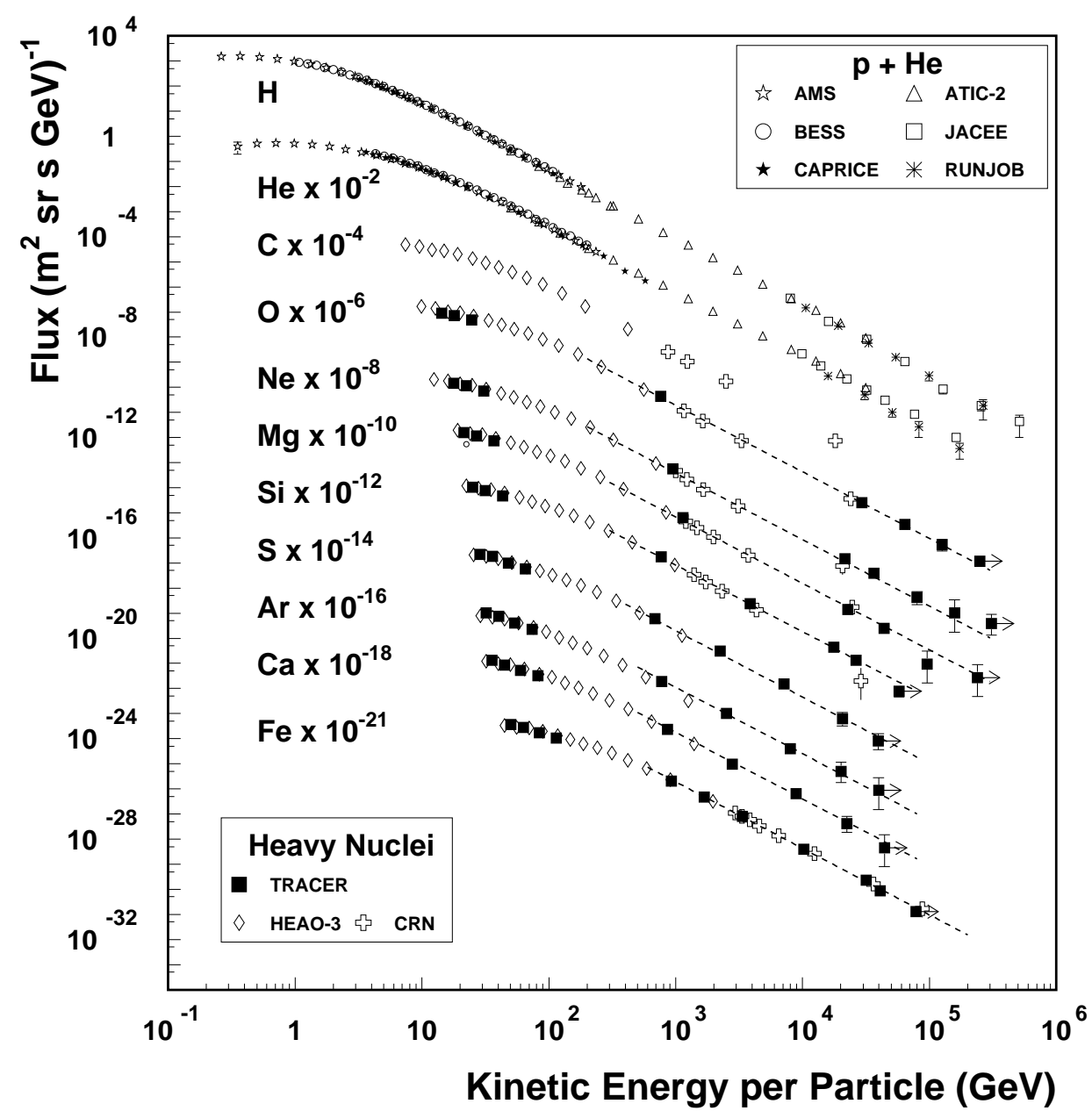

Fig. 6. Flux as a function of energy for the major components of the primary cosmic radiation. The measurements by the TRACER experiment are represented by the solid squares for the elements $\mathrm{O}, \mathrm{Ne}, \mathrm{Mg}, \mathrm{Si}, \mathrm{S}, \mathrm{Ar}, \mathrm{Ca}$, and, Fe. The dashed line represents a power-law fit to the TRACER data above $20 \mathrm{GeV} a m u^{-1}$. For references to the data presented in this plot see [7]-[16] and references therein.

\section{Resulting Energy Spectra}

The energy spectra, in terms of absolute intensities at the top of the atmosphere, for the elements $\mathrm{O}, \mathrm{Ne}, \mathrm{Mg}, \mathrm{Si}, \mathrm{S}, \mathrm{Ar}, \mathrm{Ca}$, and Fe are plotted as solid squares in Figure 6. For clarity, the intensity of each element is scaled by a factor shown on the left. Existing data from measurements in space with HEAO-3 (open diamonds [8]) and CRN (open crosses [9]) are shown for comparison. For completeness, data on the light primary cosmic ray components (protons, helium, and carbon) which are not measured with TRACER are also included. These data come from measurements 
in space (AMS [10]) and on balloons (ATIC [11, BESS [12, 13], CAPRICE [14, JACEE [15, RUNJOB [16]).

Note the large range in intensity (10 decades) and particle energy (4 decades) covered by TRACER. As can be seen, the energy spectra for $\mathrm{O}, \mathrm{Ne}, \mathrm{Mg}$, and $\mathrm{Fe}$ extend up to and beyond $10^{14} \mathrm{eV}$ particle ${ }^{-1}$. The energy range is limited by the current exposure and not by the saturation of the TRD. No significant change in spectral slope is evident at the highest energies. The energy spectrum of each element, as measured by TRACER, can be fit to a power law above $20 \mathrm{GeV} \mathrm{amu}^{-1}$ (dashed line in Figure 6). The resulting spectral indices are remarkably similar with an average exponent of $-2.65 \pm 0.05$.

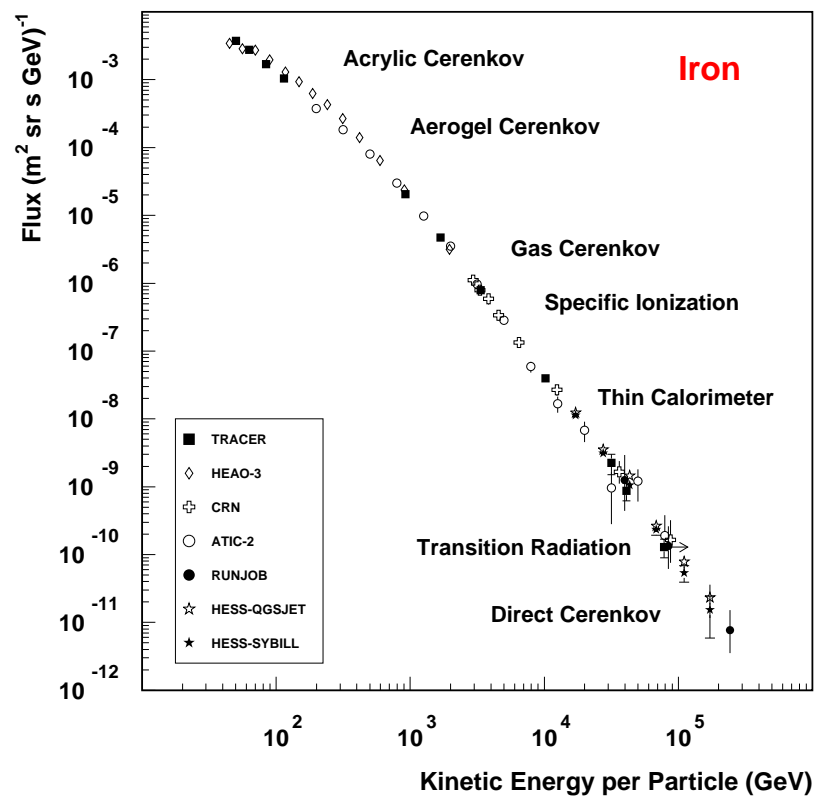

Fig. 7. Energy spectrum for iron nuclei highlighting the complementarity between detection techniques employed in cosmic-ray composition measurements.

Figure 7 compares the TRACER results for iron nuclei with results from previous experiments in space (HEAO-3 [8] and CRN [9]), and on balloons (ATIC-2 [11] and RUNJOB [16]). Figure 7 also illustrates the variety of detection techniques used in measuring the energy of heavy nuclei. Within the statistical uncertainties (which in some measurements are quite large), the data indicate fairly consistent results. Also presented in Figure 7 are recent results from the ground based HESS Imaging Air Cherenkov Telescope using the Direct Cherenkov Technique [17. Here, two flux values are presented for each energy arising from ambiguities from different nuclear interaction models used in the data analysis [18. Again, the HESS results 
are consistent with TRACER.

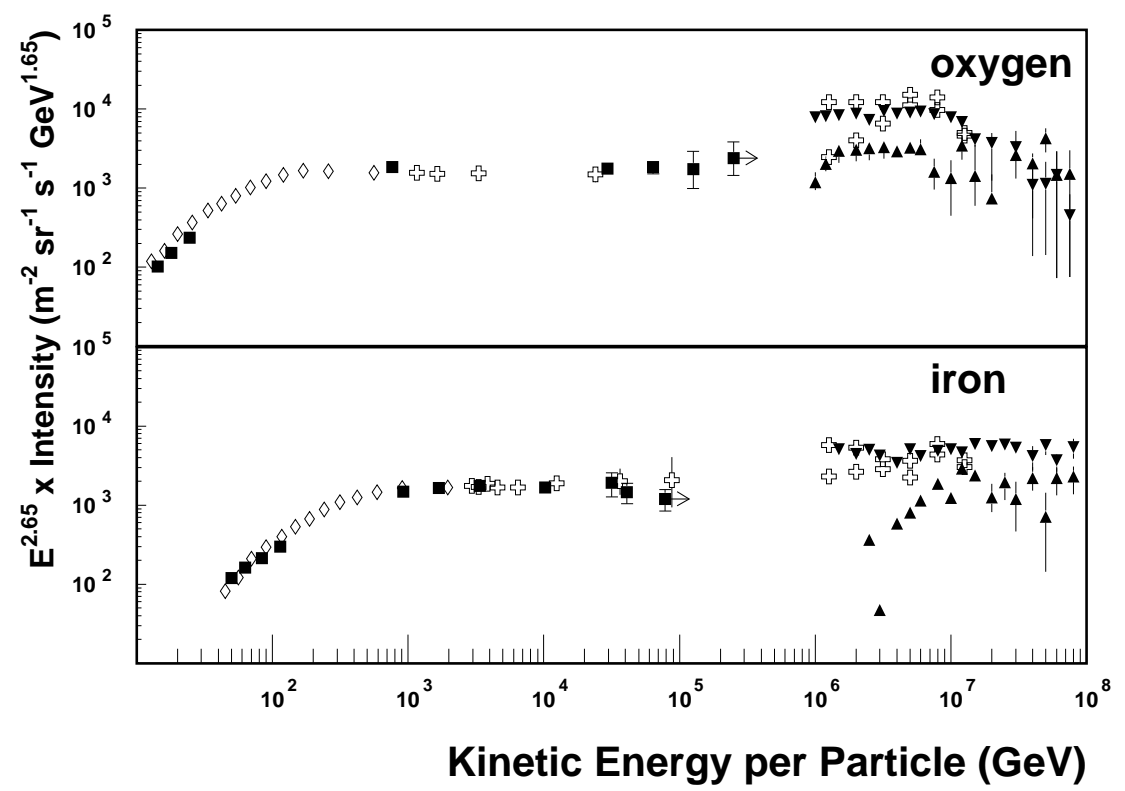

Fig. 8. Energy spectra multiplied by $\mathrm{E}^{2.65}$ from TRACER (solid squares) compared with the interpretation of air-shower data of KASCADE (filled triangles, for two different interaction models: Antoni et al. (2005)) and of EAS-TOP (open crosses, two data points for each energy represent upper and lower limits: Navarra et al. (2003). [From Ave et al.]

\subsection{Comparison with Air-shower Data}

Data for oxygen and iron nuclei from TRACER are compared in Figure 8 with energy spectra derived from indirect air-shower observations of the EAS-TOP collaboration [19] and of the KASCADE group [20. These experiments report results for groups of elements and not individual elements; therefore the fluxes for the "CNO group" probably have about twice the intensity than oxygen alone while the "iron group" is probably dominated by iron. Furthermore, these results remain ambiguous as they depend strongly on the choice of the nuclear interaction model used in the analysis. The TRACER results do not yet overlap with the energy region of the air-shower data. Additional measurements with TRACER should help to close the gap and to provide significant constraints on the interpretation of air-shower results. 


\section{Future Measurements with TRACER}

Extending the energy spectra of the heavy elements towards $10^{15} \mathrm{eV}_{\text {particle }}{ }^{-1}$ is an ongoing goal of TRACER. However, to determine the energy spectra and relative abundances for each nuclear species at the cosmic-ray source, the mode of galactic propagation must be understood. The simplest model of cosmic-ray propagation assumes an equilibrium between the production of cosmic rays, from acceleration at the source or from spallation of heavier nuclei, and their loss from competing actions of escape from the Galaxy and spallation on the interstellar medium [21]. This model can be described as:

$$
N_{i}(E)=\frac{1}{\Lambda(E)^{-1}+\Lambda_{s}^{-1}}\left(\frac{Q_{i}(E)}{\beta c \rho}+\sum_{k>i} \frac{N_{k}}{\Lambda_{k \rightarrow i}}\right)
$$

where $N_{i}(E)$ is the observed intensity of element $i, \Lambda(E)$ the propagation pathlength, $\Lambda_{s}$ the spallation pathlength, $Q_{i}(E)$ the rate of production in the cosmic-ray source, $\beta=v / c, \rho$ the average density of the interstellar medium, $N_{k}$ the intensity of element $k$, and $\Lambda_{k \rightarrow i}$ the spallation mean free path for an element $k$ to spallate to element $i$.

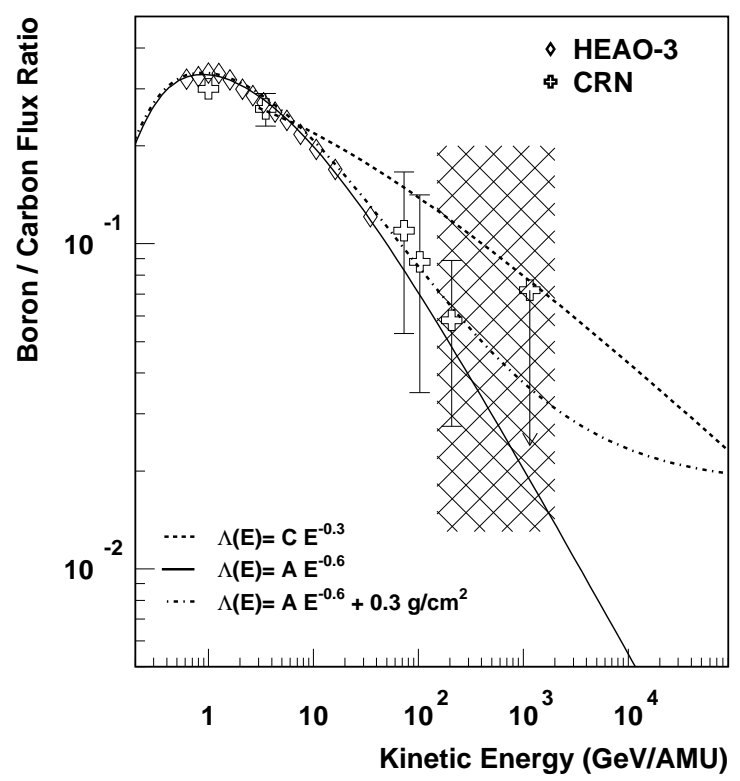

Fig. 9. Boron to carbon flux ratio with data from HEAO-3 and CRN. The solid line represent a parameterization given by Yanasak. The addition of a residual pathlength of $\Lambda_{0}=0.3 \mathrm{~g} / \mathrm{cm}^{2}$ to the Yanasak parameterization is presented by the dash-dot line. The dashed line represents a fit to $\Lambda(E) \propto E^{-0.3}$. The hashed area represents the energy region attainable from the 5-day flight of TRACER in 2006. 
The energy dependent propagation pathlength $\Lambda(E)$ can be derived from the flux ratio of secondary spallation produced particles to primary accelerated particles. This flux ratio has been observed to decrease with increasing energy [22, indicating that the mean lifetime or propagation pathlength of cosmic rays in the Galaxy decreases with energy. As an example, the boron to carbon flux ratio as measured by HEAO-3 [8] and CRN 9$]$ is shown in Figure 9. The flux ratio has been parameterized with $\Lambda(E) \propto E^{-0.6}$ [8]. However, this flux ratio has been measured accurately only to about $10^{12} \mathrm{eV}$ particle ${ }^{-1}$. It may well be that the flux ratio will eventually reach a finite asymptotic value $\Lambda_{0}$ (i.e. $\Lambda(E)=A E^{-0.6}+\Lambda_{0}$ ). The residual pathlength $\Lambda_{0}$ would represent the minimum amount of matter high-energy particles must encounter during propagation from the cosmic-ray source to Earth. Alternatively, some diffusion propagation models expect a Kolmogorov spectrum with $\Lambda(E)=C E^{-0.3}$ (see 24] for a comprehensive review, and references therein). Figure 9 illustrates that none of these scenarios cannot be fully ruled out with the present data. An extension of the boron-carbon flux ratio was a major science goal of Flight III of TRACER in 2006. It is expected that even with the limited exposure obtained in Flight III, the measurement of the boron-carbon flux ratio will reach into the $10^{13}-10^{14} \mathrm{eV}$ particle ${ }^{-1}$ range. This energy region is represented as the shaded area in Figure 9.

The flux ratio of the secondary sub-Fe elements $(Z=21-25)$ to iron $(Z=26)$ can also be used to determine the propagation parameters $\Lambda(E)$ and $\Lambda_{0}$. The sub-Fe elements are produced by the spallation of iron nuclei during propagation. Figure 10 shows the currently available energy spectra for the sub-Fe elements along with B, $\mathrm{C}, \mathrm{Ar}$, and Ca spectra. The energy spectra for the sub-Fe elements do not yet reach $10^{12} \mathrm{eV}$ particle ${ }^{-1}$.

TRACER is unable to measure the energy spectra for the sub-Fe elements with the present detector configuration. The main reasons for this are the limited charge resolution, due to nonlinearity of the scintillator response at high charges, and the limited photo-electron statistics of the Cherenkov counter. It is therefore proposed to replace one of the acrylic Cherenkov counters with a counter containing an aerogel radiator (refractive index 1.04), and to use a more efficient readout system for the second acrylic Cherenkov counter. For relativistic particles the charge would then be reconstructed using the acrylic and the aerogel Cherenkov counters. Contrary to the behavior of the scintillators, both signals are strictly proportional to $Z^{2}$ and the charge resolution would be independent of charge. This technique has been successfully utilized in the balloon borne Trans Iron Galactic Recorder (TIGER) instrument 25], which achieved a charge independent resolution of 0.22 charge units. Monte-Carlo simulations have shown that a charge resolution of 0.2 charge units could be achieved with the proposed Cherenkov counter system [26 27. Figure 11 shows the results from a simulated charge reconstruction for such a setup. The left-hand panel shows a cross correlation plot of the simulated signals from the aerogel and acrylic counters. The vertical lines represent cosmic rays of constant 


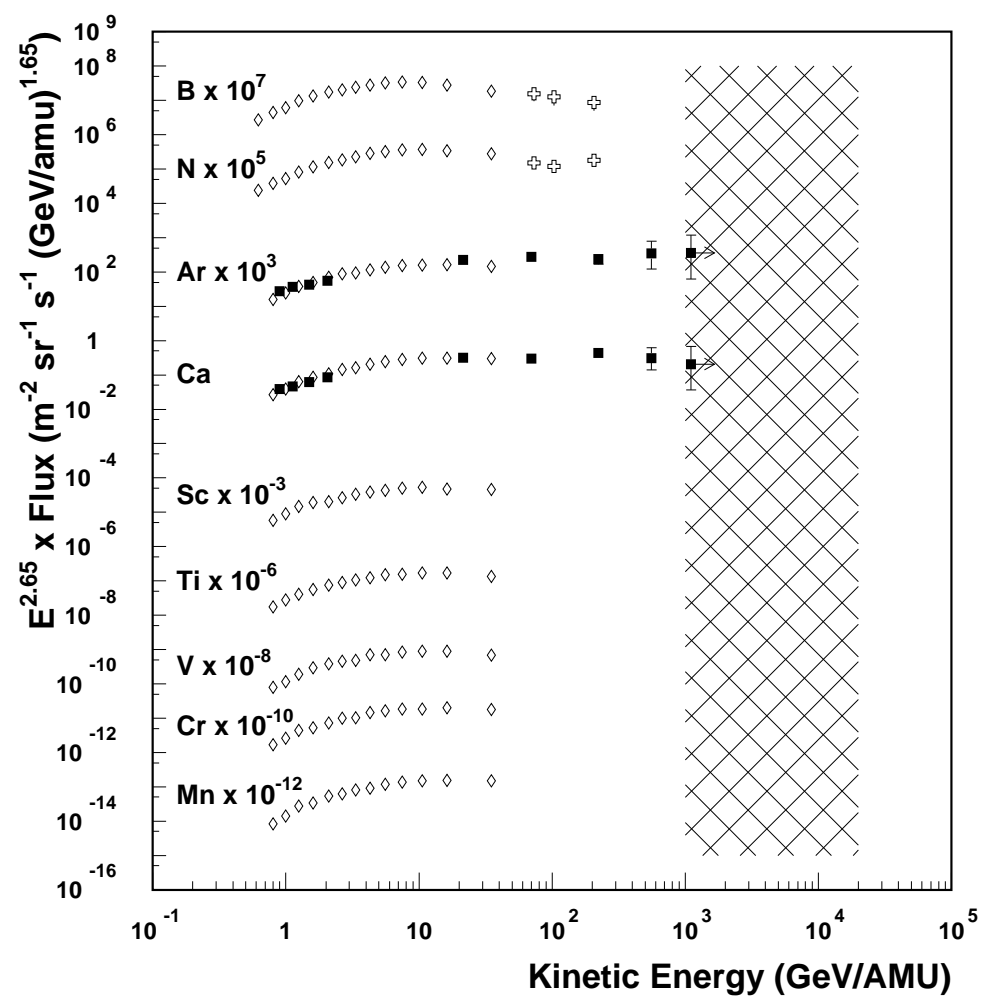

Fig. 10. Energy spectra multiplied by $E^{2.65}$ of purely secondary elements (B and sub-Fe) and mixed elements (primary and secondary: N, Ar, and Ca) from the HEAO-3 (open diamonds), CRN (open crosses) and TRACER (solid squares). The hashed region represents the energy region attainable with a 30 day flight of the upgraded TRACER.

charge. The resulting charge histogram, shown in the right-hand panel of Figure 11, indicates that the sub-Fe elements are clearly resolved. As Antarctic flights of 30 days duration are now becoming a reality [28, such an exposure of the upgraded TRACER instrument would extend the energy spectra for all secondary elements to $10^{14} \mathrm{eV}$ particle ${ }^{-1}$. This energy region is indicated by the shaded region in Figure 10 .

\section{Conclusions}

The TRACER program has pioneered the development of a TRD using proportional tube arrays to directly measure the energy spectra of cosmic-ray nuclei. The coupling of the TRD with the Cherenkov and DEDX counters enables an energy measurement of individual nuclei across 5 decades in energy and provides a technique to uniquely identify the rare high-energy particles with a discrimination power of $>10^{4}$.

TRACER has measured the energy spectra of the elements $\mathrm{O}, \mathrm{Ne}, \mathrm{Mg}, \mathrm{Si}, \mathrm{S}$, $\mathrm{Ar}, \mathrm{Ca}$, and $\mathrm{Fe}$ up to $10^{14} \mathrm{eV}$ particle ${ }^{-1}$. This data set currently represents the most comprehensive measurements to date of heavy nuclei with individual charge 

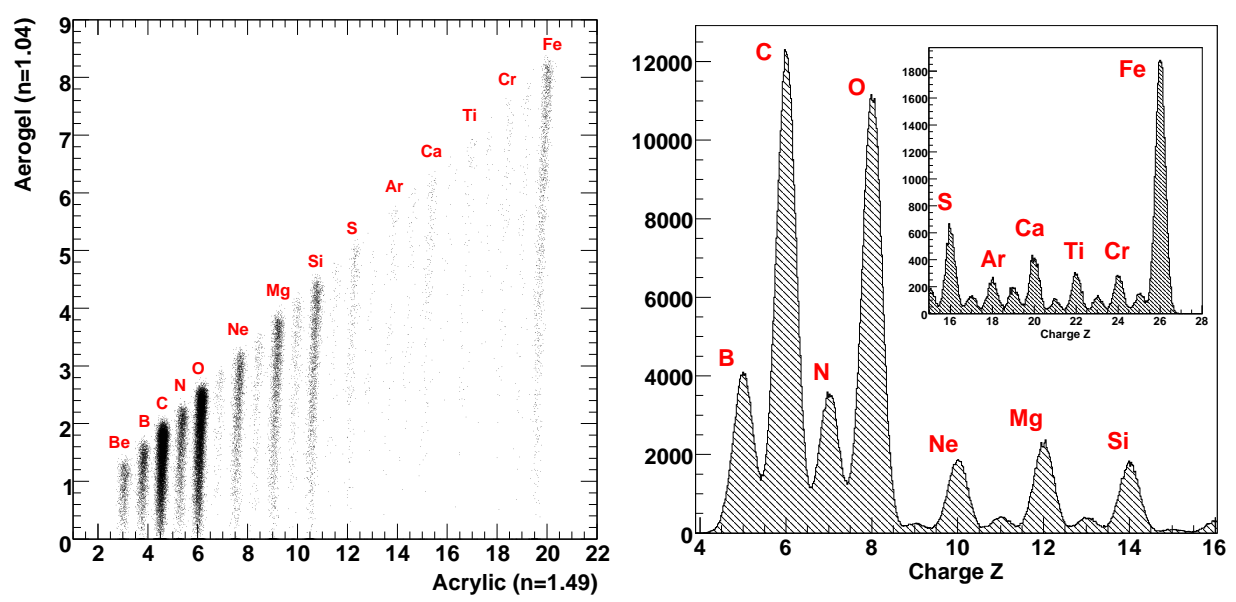

Fig. 11. (Left-hand panel) Cross correlation plot of simulated signals from two Cherenkov counters; one containing an aerogel radiator (refractive index $=1.04$ ) and the second an acrylic plastic radiator (refractive index $=1.49$ ). (Right-hand Panel) Resulting charge histogram with charge resolution of 0.2 charge units.

resolution. The measured energy spectra reach similar power laws at high energies and no change in slope is evident. The data indicate a common origin and mode of propagation for all elements and support the SNR theory of the origin of galactic cosmic rays.

TRACER has been upgraded to include a measurement of the secondary to primary flux ratio. Data analysis of the boron to carbon flux ratio is currently underway. Future upgrades of TRACER will include measurements of the sub-Fe elements.

\section{Acknowledgments}

This work has been made possible by the enormous contributions of the TRACER team: Maximo Ave, Florian Gahbauer, Christian Höppner, Jörg Hörandel, Masakatsu Ichimura, Jesse Marshall, Dietrich Müller, Andreas Obermeier, and Andrew Romero-Wolf. We gratefully acknowledge the services of the University of Chicago Engineering Center in particular Gary Kelderhouse, Gene Drag, Casey Smith, Richard Northrop and Paul Waltz. We thank the staff of the Columbia Scientific Balloon Facility, the NSF Antarctic Program and the Swedish Space Corporation for support during the balloon campaigns. This work was supported by NASA grants NAG55305, NN04WC08G and NNG06WC05G. Numerous students have participated in the construction and refurbishment of the instrument under support from the Illinois Space Grant Consortium. 


\section{References}

1. J. Cronin, T.K. Gaisser \& S.P. Swordy, Scientific American 276, 44 (1997)

2. G. Hartmann, D. Müller, T. Prince, Physical Review Letters 38, 1368 (1977)

3. R. Bellotti et al., Nucl. Phys. B, Proc. Suppl. 54B, 375 (1997)

4. S.W. Barwick et al., AP J 482L, 191B (1997)

5. J. L'Heureux, J. Grunsfeld, P. Meyer, D. Müller, \& S.P. Swordy, NIM A 295, 246 (1990)

6. F. Gahbauer et al., AP J 607, 333 (2003)

7. M. Ave et al., AP J 678, 262 (2008)

8. J.J. Engelmann et al., Astronomy \& Astrophysics 233, 96 (1990)

9. D. Müller et al., $A P J$ 374, 356 (1991)

10. AMS Collaboration, Physics Letters B490, 27 (2000)

11. H.S. Ahn et al., Advances in Space Science Reviews 37, 1950A (2006)

12. T. Sanuki et al., AP J 545, 1135 (2000)

13. S. Haino et al., Physics Letters B594, 35H (2004)

14. M. Boezio et al., Astroparticle Physics 19, 583 (2003)

15. K. Asakimori et al., AP J 502, 278 (1998)

16. V. Derbina et al., AP J 628L, 41D (2005)

17. D. Kieda et al., Proc. 27th ICRC 1, 147 (2003)

18. F. Aharonian et al., Physical Review D75, 042004 (2007)

19. G. Navarra et al., Proc. 28th ICRC 1, 147 (2003)

20. T. Antoni et al., Astroparticle Physics 24, 1 (2005)

21. S. Swordy et al., AP J 403, 658S (1993)

22. E. Juliusson et al., Phys. Rev. Lett. 29, 445 (1972)

23. N. E. Yanasak et al., AP J 563, 768

24. A. Strong, I. Moskalenko, V. Ptuskin, Annu. Rev. Nucl. Part. Sci. 57, 285-327 (2007)

25. J. T. Link et al., Proc. 28th ICRC 4, 1781L (2003)

26. J. Marshall \& A. Obermier, Private communication (2008)

27. T. Hams, Private communication (2008)

28. W.V. Jones, Inter. Cosmic-ray Conf 10, 173J (2005) 